\title{
Ultrasonographic assessment of experimentally induced gastric perforation in beagle dogs
}

\author{
Dongryun Nam ${ }^{1}$, Taesung Hwang ${ }^{2}$, Hee Chun Lee ${ }^{2, *}$ \\ ${ }^{1}$ Yangsan Animal Medical Center, Yangsan 50629, Korea \\ ${ }^{2}$ Institute of Animal Medicine, College of Veterinary Medicine, Gyeongsang National University, Jinju 52828, Korea
}

\begin{abstract}
The goals of this study were, first, to evaluate the feasibility of inducing gastric perforation with $99 \%$ alcohol injection after electrocautery (EA-method), and, second, to observe "enhanced peritoneal stripe sign (EPSS)" and other lesions upon induction of gastric perforation. Six clinically normal beagle dogs were prepared for gastric perforation using endoscopy. After gastric perforation, EPSS and other lesions on ultrasonography were observed eventually (at $0 \mathrm{~h}, 3 \mathrm{~h}$, day 1 , day 2, day 3, day 4, day 5, and day 6). We graded the EPSS depending on its width and number. EPSS was observed until day 4 of the examination in all the 6 dogs. The grades of EPSS were the highest at $3 \mathrm{~h}$ and declined gradually. Peritoneal effusion was observed in all dogs at $3 \mathrm{~h}$ and on day 1 . Regional bright mesenteric fat was confirmed in all dogs on days 3 and 4. In conclusion, gastric perforation can be induced by EA-method. EPSS and peritoneal effusion appear at a very early stage, and regional bright mesenteric fat was identified on days 3 and 4 in almost all dogs with gastric perforation.
\end{abstract}

Keywords: EPSS, gastric perforation, pneumoperitoneum, dogs

*Corresponding author

Hee Chun Lee

Institute of Animal Medicine, College of Veterinary Medicine, Gyeongsang National University, 501 Jinju-daero, Jinju 52828, Korea

Tel: $+82-55-772-2356$

Fax: +82-55-772-2330

E-mail: lhc@gnu.ac.kr

ORCID:

Dongryun Nam

https://orcid.org/0000-0001-6724-6601

Taesung Hwang

https://orcid.org/0000-0001-6730-6061

Hee Chun Lee

https://orcid.org/0000-0001-5936-9118

Conflict of Interest

There is no conflict of interest.

Received: March 19, 2019

Revised: June 4, 2019

Accepted: June 19, 2019

\section{Introduction}

Patients with acute abdominal pain are often initially screened with abdominal radiographs [1]. Detection of pneumoperitoneum is of prime importance because it represents an acute abdominal emergency requiring aggressive treatment [2]. In a previous human study, despite careful technical attention, radiologists failed to detect pneumoperitoneum [3]. Computed tomography (CT) has been shown to be more sensitive than abdominal and chest radiography in the detection of free air [4-6]. However, the detection of a small volume of pneumoperitoneum on abdominal CT may be insignificant [7]. Sonography is routinely used to examine patients with undiagnosed abdominal pain. "Dirty shadows" and reverberation artifacts associated with gas in the lumen of the gut occur whenever the ultrasound beam encounters gas in the scanning field, which is considered a normal observation on abdominal sonography. However, the identification of gas in the peritoneal cavity or retroperitoneum is strongly associated with a gastrointestinal (GI) perforation [8].

In previous studies, enhanced peritoneal stripe sign (EPSS) was a reliable and specific ultrasonographic sign for the diagnosis of pneumoperitoneum, and small amounts of peritoneal free gas above $0.4 \mathrm{~mL}$ were detected in dogs during ultrasonography $[9,10]$. However, these studies were based on air injection into the peritoneal cavity. Because the literature has limited information involving dogs with naturally-occurring pneumoperitoneum, we investigated a method of gastric perforation induction similar to clinical practice. The purposes of this study were, first, to evaluate the feasibility of electrocautery (EA)-method, and, second, to observe aspects of EPSS and other lesions following gastric perforation.

\section{Materials and Methods}

This study includes 6 clinically healthy beagle dogs (4 males and 2 females). Body weight ranged from 8.0 to $13.8 \mathrm{~kg}$ (aged between 3 and 5 years). The dogs showed no clinical history or abnormal signs. Animal care 
and experiments were carried out in compliance with A Guide for the Care and Use of Laboratory Animals, published by Gyeongsang National University (GAR-101118-X0010).

All dogs were preanesthetized with glycopyrrolate 0.01 $\mathrm{mg} / \mathrm{kg}(0.2 \mathrm{mg} / \mathrm{mL}$; Myungmoon, Korea) given subcutaneously and general anesthesia (Isoflurane 100\% liquid; Kyongbo, Korea) was induced by propofol $6 \mathrm{mg} / \mathrm{kg}(10 \mathrm{mg} / \mathrm{mL}$, Myungmoon). Using an endoscope (CV-150 GIF XQ240; Olympus, Japan), the stomach was inflated under inhalation anesthesia. Avoiding injury to blood vessels, the wall of greater curvature was damaged with an electrosurgical knife (RH-2000 Plus; Visco, Korea) followed by injection of $99 \%$ ethyl alcohol $1.0 \mathrm{~mL}$ into the damaged spot using 23 gauge endoscopic injection needle (NM-401L-0423; Olympus). The procedures were carried out with care to ensure that the damage did not directly penetrate the gastric serosa. After the experimental injury, intragastric gas was eliminated.

The ultrasound machine $\left(\right.$ Xario $^{\circledR}$ SSA-660A; TOSHIBA, Japan) was used with a $12.0 \mathrm{MHz}$ linear transducer. The abdomen was prepared by clipping the hair over the entire ventral aspect of the abdomen. Coupling gel was applied to the skin. The dogs were scanned in dorsal recumbency. All the ultrasonographic procedures were performed by the same examiner who was an expert in sonographic examination. EPSS and other lesions were evaluated depending on the time set ( 0 h, $3 \mathrm{~h}$, day 1 , day 2 , day 3 , day 4 , day 5 , and day 6) after EA-method. The "other lesions" were categorized according to a previous study: regional bright mesenteric fat, peritoneal effusion, fluid-filled stomach or intestines, GI wall thickening, loss of GI wall layering, regional lymphadenopathy, reduced GI motility, pancreatic changes, corrugated intestines, presence of a mass, presence of a foreign body, and gastric wall mineralization [8]. The EPSS grade was as follows: I (non-observed), II (the width of EPSS less than 5 $\mathrm{mm}$ ), III (the width of EPSS between 5 and $10 \mathrm{~mm}$ or more than EPSS of grade II), and IV (the width of EPSS longer than $10 \mathrm{~mm}$ ) (Table 1 and Fig. 1).

The grades of EPSS were statistically analyzed using Kruskal-Wallis test at a significance level of 0.05. The MannWhitney test was used to compare the EPSS grade at $0 \mathrm{~h}$ with grades at other times, and a $p$ value of $<0.00176$ was considered significant in Mann-Whitney test. All statistical analyses were performed using the SPSS software (version 14.0; SPSS Inc., USA).

\section{Results}

The EPSS was observed for the first time at $3 \mathrm{~h}$ after injury in all the 6 dogs. Subsequently, the EPSS grade was gradually decreased over time (Table 2 ). There was a highly significant difference in grades of EPSS at different time intervals $(p<0.001)$. Highly significant differences were observed in EPSS grades between $0 \mathrm{~h}$ and $3 \mathrm{~h}(p=0.0009)$, and between $0 \mathrm{~h}$ and day $2(p=0.0009)$ (Table 3$)$.

Other lesions identified in this study included regional bright mesenteric fat, peritoneal effusion, fluid-filled stomach or intestines, GI wall thickening, regional lymphadenopathy, reduced GI motility, and pancreatic changes (Table 4). Peritoneal effusion was observed in all dogs at $3 \mathrm{~h}$ and on day 1. Regional bright mesenteric fat was confirmed in all dogs on days 3 and 4 .

Table 1. Grades of EPSS

\begin{tabular}{ll}
\hline \hline Grade & Width of EPSS \\
\hline I & Non-observed \\
II & $\leq 5 \mathrm{~mm}$ \\
III & $>5$ to $\leq 10 \mathrm{~mm}$ \\
IV & $>10 \mathrm{~mm}$ \\
\hline
\end{tabular}

EPSS, enhanced peritoneal stripe sign.

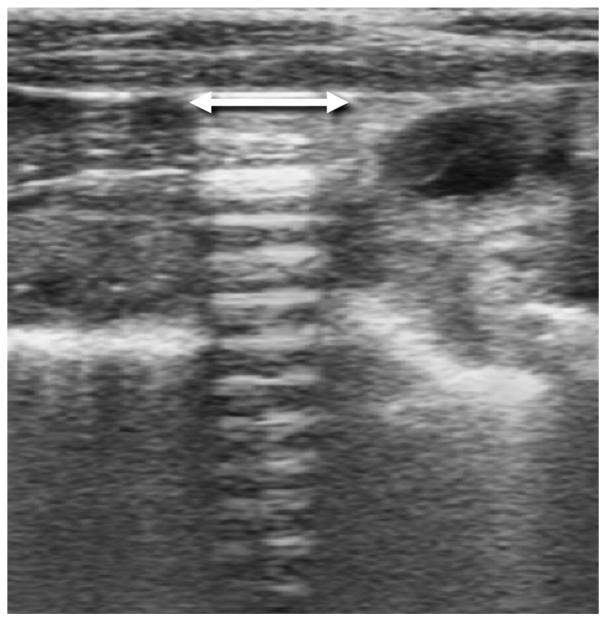

Fig. 1. Width of EPSS (arrow).

EPSS, enhanced peritoneal stripe sign.

Table 2. Grades of EPSS at different time intervals

\begin{tabular}{llllccccc}
\hline \hline & 0 h & 3 h & Day 1 & Day 2 & Day 3 & Day 4 & Day 5 & Day 6 \\
\hline Case 1 & I & IV & III & III & II & I & I & I \\
Case 2 & I & IV & IV & III & III & I & I & I \\
Case 3 & I & IV & IV & III & III & III & I & I \\
Case 4 & I & IV & III & III & II & I & I & I \\
Case 5 & I & IV & IV & III & III & II & I & I \\
Case 6 & I & IV & III & III & I & I & I & I \\
\hline
\end{tabular}

EPSS, enhanced peritoneal stripe sign. 
Table 3. Comparison of EPSS grade at $0 \mathrm{~h}$ with that at other times

\begin{tabular}{cccccccc}
\hline \hline & \multicolumn{7}{c}{ Mann-Whitney test } \\
\hline $0 \mathrm{~h}$ & $3 \mathrm{~h}$ & Day 1 & Day 2 & Day 3 & Day 4 & Day 5 & Day 6 \\
\hline
\end{tabular}

Values are presented as $p$ value.

EPSS, enhanced peritoneal stripe sign.

There were highly significant differences in EPSS grades between ${ }^{*} 0 \mathrm{~h}$ and $3 \mathrm{~h}$, and between ${ }^{\dagger} 0 \mathrm{~h}$ and day 2.

Table 4. The number of dogs which were shown specific findings during ultrasonographic examination after experimentally induced gastric perforation

\begin{tabular}{lcccccccc}
\hline \hline & $0 \mathrm{~h}$ & $3 \mathrm{~h}$ & Day 1 & Day 2 & Day 3 & Day 4 & Day 5 & Day 6 \\
\hline Regional bright mesenteric fat & 0 & 0 & 4 & 5 & 6 & 6 & 5 & 5 \\
Peritoneal effusion & 0 & 6 & 6 & 4 & 4 & 4 & 4 & 4 \\
Fluid-filled stomach or intestines & 0 & 3 & 1 & 1 & 1 & 1 & 0 & 0 \\
GI wall thickening & 0 & 1 & 3 & 3 & 4 & 3 & 3 & 3 \\
Regional lymphadenopathy & 0 & 0 & 0 & 2 & 3 & 3 & 3 & 2 \\
Reduced GI motility & 0 & 0 & 0 & 1 & 0 & 0 & 0 & 0 \\
Pancreatic changes & 0 & 0 & 3 & 3 & 3 & 3 & 3 & 1 \\
\hline
\end{tabular}

GI, gastrointestinal.

\section{Discussion}

Ultrasonography is commonly used for the detection of pneumoperitoneum in clinical practice [11]. A pneumoperitoneum is recognized by the sonographic appearance of EPSS $[9,12]$. EPSS and other lesions were not observed in case of damaged gastric wall following electrocautery alone or injection of $99 \%$ alcohol in pilot studies. EPSS and other lesions, however, were identified for the first time at $3 \mathrm{~h}$ after EAmethod, which suggests that EA-method failed to result in direct penetration of the gastric wall, but induced a gastric perforation within $3 \mathrm{~h}$.

EPSS is associated with artifacts at the air-soft tissue interface, between the nondependent abdominal wall and intraabdominal structures such as the liver, stomach, omentum, or intestine $[9,12]$. Increasing the amount of intraperitoneal gas increased the width of EPSS as well. Under this assumption, we graded EPSS according to its width. In this study, the EPSS grades were the highest at $3 \mathrm{~h}$ of examination in all dogs. And the EPSS grades declined gradually with time, no EPSS was observed after day 5. We thought that the EPSS decreased due to spontaneous healing for gastric perforation. We made gastric perforation by experimentally which is very small and no further irritation. Therefore, self-healing may have occurred. In addition, peritoneal effusion was identified at $3 \mathrm{~h}$ of examination in all dogs, and the number of dogs with peritoneal effusion decreased after the second day examination. Regional bright mesenteric fat was confirmed in all dogs on days 3 and 4, suggesting that EPSS and peritoneal effusion appeared at the very early stages of gastric perforation, followed by regional bright mesenteric fat.

As described above, the EPSS grade at $0 \mathrm{~h}$ was grade I, which was normal during ultrasonographic examination. The
EPSS grade at $0 \mathrm{~h}$ was significantly different from those at $3 \mathrm{~h}$ and on day $2(p<0.0017)$. The differentiation in the grade between $0 \mathrm{~h}$ and day 1 was nearly significant $(p=0.0018)$. We assume that the small number of dogs used in this experiment may contribute to this gap.

Based on our study results, gastric perforation can be induced with EA-method. EPSS and peritoneal effusion appear at very early stages, and regional bright mesenteric fat may be detected on days 3 and 4 in almost all dogs with gastric perforation.

\section{References}

1. Miller RE, Nelson SW. The roentgenologic demonstration of tiny amounts of free intraperitoneal gas: experimental and clinical studies. Am J Roentgenol Radium Ther Nucl Med 1971;112:574-585.

2. Paster SB, Brogdon BG. Roentgenographic diagnosis of pneumoperitoneum. JAMA 1976;235:1264-1267.

3. Roh JJ, Thompson JS, Harned RK, Hodgson PE. Value of pneumoperitoneum in the diagnosis of visceral perforation. Am J Surg 1983;146:830-833.

4. Glazer GM, Buy JN, Moss AA, Goldberg HI, Federle MP. CT detection of duodenal perforation. AJR Am J Roentgenol 1981;137:333-336.

5. Jeffrey RB, Federle MP, Wall S. Value of computed tomography in detecting occult gastrointestinal perforation. $\mathrm{J}$ Comput Assist Tomogr 1983;7:825-827.

6. Phatak MG, Frank SJ, Ellis JJ. Computed tomography of bowel perforation. Gastrointest Radiol 1984;9:133-135.

7. Bulas DI, Taylor GA, Eichelberger MR. The value of CT in detecting bowel perforation in children after blunt abdominal trauma. AJR Am J Roentgenol 1989;153:561-564.

8. Boysen SR, Tidwell AS, Penninck DG. Ultrasonographic findings in dogs and cats with gastrointestinal perforation. 
Vet Radiol Ultrasound 2003;44:556-564.

9. Choi H, Lee Y, Park K, Yeon S, Lee H. Sonographic detection of small amounts of free peritoneal gas in beagle dogs. J Vet Med Sci 2012;74:491-494.

10. Kim SY, Park KT, Yeon SC, Lee HC. Accuracy of sonographic diagnosis of pneumoperitoneum using the EPSS in beagle dogs. J Vet Sci 2014;15:195-198.
11. Lee DH, Lim JH, Ko YT, Yoon Y. Sonographic detection of pneumoperitoneum in patients with acute abdomen. AJR Am J Roentgenol 1990;154:107-109.

12. Asrani A. Sonographic diagnosis of pneumoperitoneum using the 'enhancement of the peritoneal stripe sign.' A prospective study. Emerg Radiol 2007;14:29-39. 